\title{
Detection of Tendon Tears by Degree of Linear Polarization Imaging
}

\author{
Jihoon Kim \\ Biomedical Engineering, Northwestern University, Evanston, IL 60208, USA \\ Junghwan Oh \\ Mechanical Engineering, Pukyong National University, San 100, Yongdang-dong, Nam-gu, \\ Busan 608-739, Korea \\ Hyun Wook Kang \\ Innovation Center, American Medical Systems, Inc., San Jose, CA 95134, USA \\ Ho Lee \\ School of Mechanical Engineering, Kyungpook National University, 1370 Sankyuk-dong, \\ Buk-gu, Daegu 702-701, Korea \\ Jeehyun Kim* \\ School of Electrical Engineering and Computer Science, Kyungpook National University, $13 \% 0$ \\ Sankyuk-dong, Buk-gu, Daegu 702-701, Korea
}

(Received September 15, 2009 : revised October 13, 2009 : accepted October 14, 2009)

\begin{abstract}
A Stokes polarimetry imaging (SPI) system was developed and utilized to detect tendon tears by constructing the degree of linear polarization (DOLP) image maps after linearly polarized light illumination. The micro and partial-thickness tears of turkey tendons were made and imaged by the SPI system at different incident polarization angles (IPA) with different mechanical loads on the tendon. The micro and partial-thickness tendon tears were detected by the DOLP images due to weak birefringence around the tears. The tendon tears were detected by a highest DOLP contrast at longest visible wavelength (Red, $650 \pm 50 \mathrm{~nm}$ ). All polarized images showed modulated DOLP as the incident polarization angle (IPA) was varied. The varying DOLP allowed the optimal detection of the micro and partial-thickness tendon tears at a certain IPA. The SPI system with variable IPA and spectral information can improve the detection of the tendon tears by higher visibility of fiber orientations, and thereby improve diagnosis and treatment of the tendon related injuries.
\end{abstract}

Keywords: Polarization, Tendon, Tear, Imaging, CCD

OCIS codes : (110.4500) Optical coherence tomography; (170.0170) Medical optics and biotechnology; (120.3890) Medical optics instrumentation

\section{INTRODUCTION}

The rotator cuff is the group of muscles and their tendons that stabilize a human shoulder. The tendons of the rotator cuff have an important role to rotate arms by connecting the scapula to the humerus. A rotator cuff tear is the serious disease of a professional athlete who makes a movement of throwing at its maximum. It leads to pain and restricted movement of

*Corresponding author: jeehk@knu.ac.kr the arm. The rupture of the rotator cuff tendons is mostly caused by gradual and progressive histopathologic changes [1] and the accurate measurement of the level of a rotator cuff tear is important [2].

Arthroscopy has been used as a gold standard to measure the level of a rotator cuff tear. However, an arthroscopic evaluation of the rotator cuff has been difficult due to the limitation of access to the tendon with a restricted field of view [2]. Recently, MRI and ultrasonography have been used as non-invasive methods 
for the detection of rotator cuff tears. However, they are limited due to the low sensitivity [3] and the arthroscopic assessment should be followed.

Detection of the optical properties of tissue has been emphasized to diagnose human diseases [4-6]. The polarization property of tissue has been detected for imaging objects inside of the scattering media such as tissue [7]. Polarization imaging can provide tissue type information and depolarization of polarized light in human tissue depends on tissue type [8]. The bundle of collagen fibers in tendon is strongly form-birefringent and the collagen fiber orientation of the tendon can be detected by a polarization imaging system [9]. Additionally, in this study, RGB filters for an incoming polarized light are added to allow spectral polarization imaging and spectral imaging can provide information deep in tissue $[10,11]$.

In this study, we developed a Stokes-polarimetry imaging system and examined it for the detection of different types of tendon tears for the diagnosis of a rotator cuff tear.

\section{MATERIAL AND METHOD}

\section{Stokes polarimetry imaging (SPI) system}

The incident light was polarized by the linear polarizer (Colorlink) and the incident polarization angle (IPA) of the polarized light was controlled and varied from $0^{\circ}$ to $170^{\circ}$ by the motorized rotation stage (URS75CC, Newport) that holds the linear polarizer. The schematic diagram of the SPI system was shown in Figure 1.

The remitted polarized light was focused via the lens (focal length $=8 \mathrm{~cm}$, Canon EF 28-105 mm, Canon) and detected by the charge-coupled device (CCD) camera (ES4020, Redlake, 12 bit, $2048 \times 2048$ pixels,

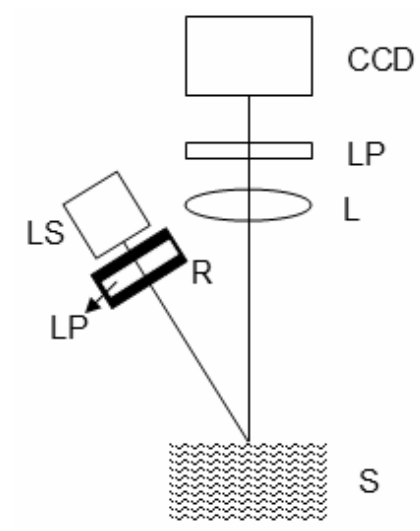

FIG 1. The schematic of the Stokes polarimetry imaging system (CCD: CCD camera, S: sample, LS: light source, LP: linear polarizer, L: lens, R: rotator). It is noted that the incident polarization angle was controlled and varied by the motorized $\operatorname{rotator}(\mathrm{R})$. resolution: $20 \mu \mathrm{m} /$ pixel). The intensities of the polarized light with $0, \pm 45, \pm 90$ were obtained from the detected intensity of the remitted light and used to calculate the linear components of the Stokes parameters (I, Q and U). The circular component of the Stokes parameters was not considered in this study due to the weak circularly polarized light from the tendon. The Stokes parameters were calculated by the following equations using the detected intensities.

$$
\begin{aligned}
& \mathrm{I}=\mathrm{I}_{0}+\mathrm{I}_{90} \\
& \mathrm{Q}=\mathrm{I}_{0}-\mathrm{I}_{90} \\
& \mathrm{U}=\mathrm{I}_{45}-\mathrm{I}_{-45}
\end{aligned}
$$

where I0, I45, I-45, and I90 represent the light intensity with linear polarization at $0^{\circ}, 45^{\circ},-45^{\circ}$ and $90^{\circ}$, respectively.

The degree of linear polarization (DOLP) was constructed by using three linear Stokes parameters (I, Q and U)

$$
\text { DOLP }=\frac{\sqrt{\mathrm{Q}^{2}+\mathrm{U}^{2}}}{\mathrm{I}}
$$

\section{Tissue preparation}

The turkey legs were obtained locally and the tendons were extracted from the legs for the polarization imaging. Each tendon was stored in a phosphate-buffered saline (PBS, PH 7.4) solution prior to the imaging and used within 24 hours.

The micro-tears were made by applying tensile force to the tendon by a hardness tester (Rocwell 2000, Instron, USA). Tensile stress was increased by a rate of $100 \mathrm{KPa} / \mathrm{s}$ until the tensile force reached 20,30 or $40 \mathrm{MPa}$ to generate the micro-tears. The length, thickness and width of each tendon were measured for the tensile stress. Rough sand papers were glued (Super glue, cyanoacrylate cement) to each end of the tendon before stretching. The $40 \mathrm{MPa}$ was the maximum tensile force without breaking the bonding by the Super glue. The tendons were embedded in a gelatin solution (Knox, Parsippany, New Jersy; 5.93 g powdered gelatin in $100 \mathrm{ml}$ of Water) to prevent the dehydration of the tendon.

The three different cuts (diagonal, horizontal and vertical) were made on the tendon by a surgical blade to imitate partial thickness tears. The cuts were shallow so as not to cut the tendon entirely.

\section{Imaging procedure}

The linearly polarized light at various incident polarization angles (IPA) illuminated the turkey tendon with micro and partial thickness tears. The IPA was rotated from $0^{\circ}$ to $170^{\circ}$ by $10^{\circ}$ increments. It took 
about 20 second to get all images at 18 different IPA. The field of view is $4 \mathrm{~cm} \times 4 \mathrm{~cm}$. All the measurements were conducted in a dark room. The DOLP image maps for the tendon were obtained with 3 different visible light filters (Red, Blue, and Green) and compared to the white light image maps.

The tendon was stretched by different loads to imitate the normal stretching of the tendon and to investigate the effect of load to the tendon polarization property in terms of DOLP. The load was controlled and varied from 0 to $2.5 \mathrm{Kg}$ by $500 \mathrm{~g}$. The dehydration of the tendon was prevented and excessive PBS on a tendon surface was wiped. The DOLP image maps at each load were imaged by the SPI system.

\section{RESULTS}

\section{Stokes polarimetry images}

\section{A. Micro tendon tear}

DOLP images of the turkey tendon that was pulled by various tensile stresses showed more severe microtears of the tendon with higher tensile stresses. The tendon without applying tensile force preserved its original vertical fiber orientation which was observed by the high DOLP (Figure 1-A). However, when the tendon was stretched with the 20,30 and $40 \mathrm{MPa}$ of tensile stresses, the collagen fiber bundles of the stretched tendon were damaged. The damage is shown as the horizontal wrinkles (Figure 1-B, C and D).

As the tendon was stretched by the higher tensile stress, the more extensively damaged fiber bundles were observed by the DOLP images. In detail, the damaged region at the central area of the tendon that was stretched by the tensile stress of $30 \mathrm{MPa}$ was probably due to the smaller cross section of the central area. The damage was not observed by the CCD images under white light (Figure 1-C-II). The CCD

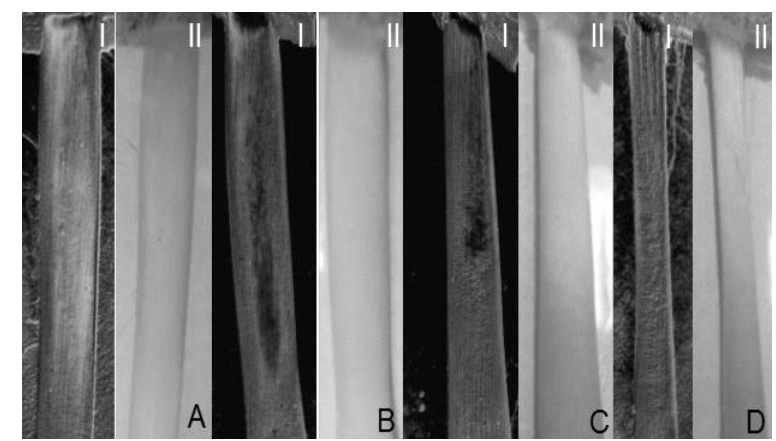

FIG 2. DOLP images (I) of the turkey tendons with different tensile stresses with comparison to the CCD images (II). (A) control, (B) $20 \mathrm{MPa}$, (C) $30 \mathrm{MPa}$ and (D) $40 \mathrm{MPa} .\left(\mathrm{IPA}=130^{\circ}\right)$. The polarized light wavelength of the DOLP images is $650 \pm 50 \mathrm{~nm}$. images did not show much tensile stress difference with comparison to the DOLP images. The DOLP images showed the orientation of the tendon collagen fibers and detected the damaged area clearly.

\section{B. Partial-thickness tendon tear}

The DOLP images of the partial-thickness tendon tear images were compared to the CCD images at the $60^{\circ}$ IPA. The polarization images showed collagen fiber orientation clearly with comparison to the CCD images. The surface wrinkles disappeared and the collagen fiber bundles of the tendon were distinguished by the higher loads. The DOLP images revealed the locations of horizontal and diagonal cuts by showing collagen fiber orientation clearly.

The effect of loading on polarization images was investigated and polarization images with different loads are shown in Figure 3. As heavier loading was applied, the wider opening of a diagonal partialthickness tear was shown. The cross-sectional collagen fiber bundles were more widely opened as the heavier load was applied. The DOLP images distinguished the tendon tears by showing the loss of birefringence in terms of the DOLP. The dark area above the partial-thickness tear (the diagonal cut in the Figure 3, DOLP images with the LP2 and LP3) may indicate the dissected collagen fiber bundles that were pulled by the tensile force at the opened area. It is noted that the size of the opened area was not changed much after the load reached to $1 \mathrm{Kg}$ (LP2). The collagen fiber bundles looked more stretched by the higher load (2 $\mathrm{Kg}$, LP3).

\section{Spectral imaging of tendon tears}

The DOLP images under the red light showed the fiber orientation with high intensity due to deeper light penetration than for other light. The dark area in the DOLP image under red light is believed to be due to the saturation of reflected light from the tendon.

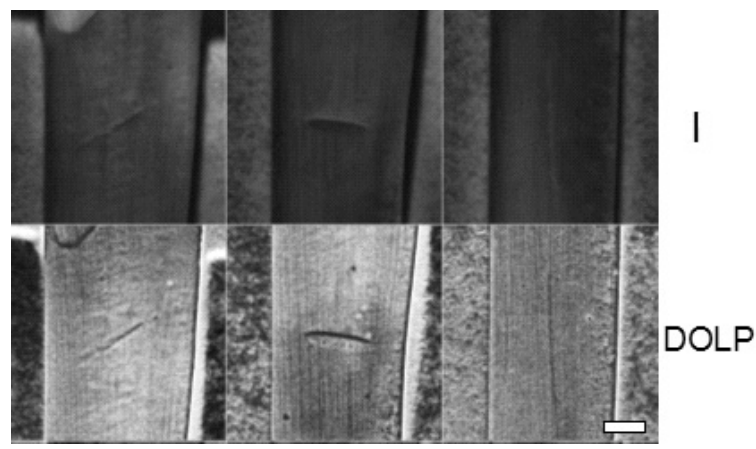

FIG 3. The DOLP images of partial thickness tears of the tendon in comparison to the CCD images (I, intensity-based). The partial thickness tears in three different directions (left: diagonal, center: horizontal, right: vertical). The IPA is $60^{\circ}$. The scale bar is $1 \mathrm{~mm}$. 
Tendon was extended by the maximum load $(2.5 \mathrm{Kg})$ and the surface of the tendon was stretched as much as possible. The polarized light illuminated a tendon with an angle and the upper part of the tendon was more reflected after being stretched. Therefore, the upper part of the cut looked darker compared to other area and it indicated the location of the cut as a result.

\section{The effect of IPA rotation}

The DOLP images (Figure 5) with varying IPA from 10 to 90 degree showed the structural information of the tendon as well as the modulation of intensity of the light reflected from the tendon. The varying IPA of incident polarized light provides better visibility to detect damages in a tendon.

\section{DISCUSSION}

\section{The detection of the micro/partial-thickness} tendon tears

The collagen fibers of the turkey tendons used in this study are regularly arranged in a longitudinal direction.

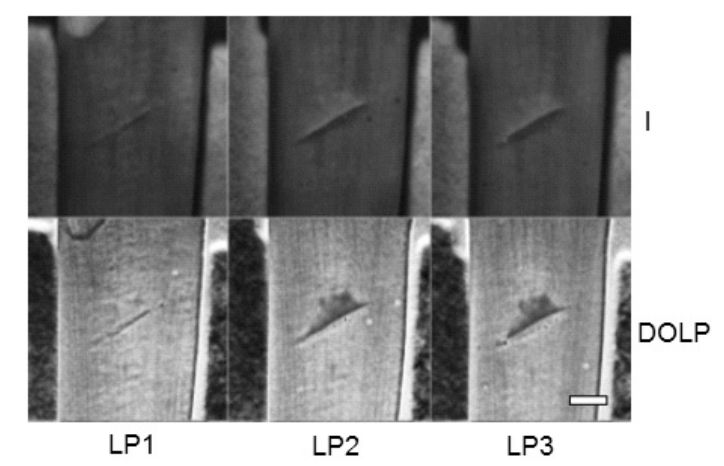

FIG. 4. The DOLP images of partial thickness tendon tears with different loads in comparison the CCD images. Three different loads (I: intensity-based CCD images, LD: Load, LD1: $0 \mathrm{Kg}$, LD2 and LD3: $1 \mathrm{Kg}$ and $2 \mathrm{Kg}$, respectively) were applied to the tendon. The IPA is $60^{\circ}$. The scale bar is $1 \mathrm{~mm}$.

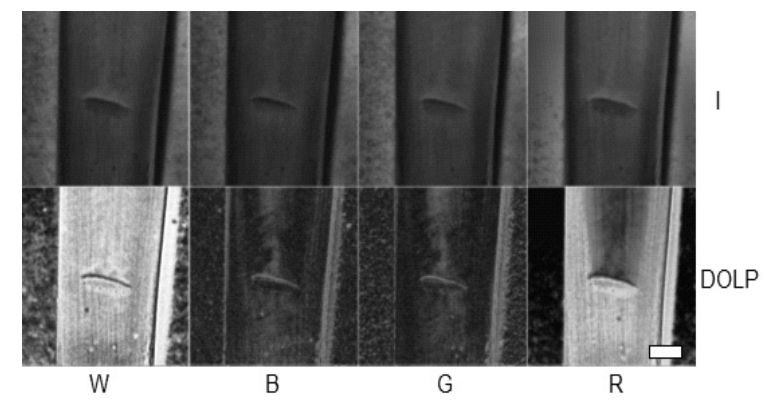

FIG. 5. Spectral imaging of the tendon, stretched by the maximum load $(2.5 \mathrm{Kg})$ : white $(\mathrm{W})$, blue $(\mathrm{B})$, green $(\mathrm{G})$ and red $(\mathrm{R})$ polarized lightilluminated on the tendon. The IPA is $60^{\circ}$. The scale bar is $1 \mathrm{~mm}$.
Tendons transmit mechanical tension forces from muscles to bones and the orientation of the collagen fibers in the tendon depends on the direction of tension exerted to the tendon [12]. The refractive index of the collagen fiber in the longitudinal direction is larger than the refractive index of the other orthogonal refractive index. This anisotropy causes the tendon linear birefringence that is the refractive index difference along the two orthogonal optical axes.

When the polarization angle of the incident polarized light was parallel to one of the optical axes of the tendon, the linear birefringence of the tendon was constant and the phase shift of incident polarized light is not significant. As a result, the DOLP is high due to the minimal depolarization. However, the orthogonal electrical fields of the incident polarized light experiences two different refractive indexes when the incident polarization angle is not parallel to one of the optical axes of the tendon. The different refractive indexes cause a phase shift relative to each other. The linear birefringence of the polarized light is defined as refractive index difference between two different orthogonal electrical fields of the light [9]. The DOLP modulation at the varying IPA (Figure 5) was due to the dependence of the DOLP on the phase shift because the phase shift is strongly related to the depolarization of the incident light.

The low DOLP at the IPAs of $40^{\circ}$ and $50^{\circ}$ (Figure 5 -D and E) indicated the significant depolarization most likely due to the loss of the tendon linear birefringence [9]. The damaged area of the tendon was shown well at the IPA of 40 to $50^{\circ}$ with comparison to the DOLP images at other IPA. The damaged tendon broke the linear birefringence of the tendon and the damaged area of the tendon was differentiated from the normal tendon. For the DOLP images (Figure 5-I) at the IPAs of $0^{\circ}$ or $90^{\circ}$, that are parallel to the one

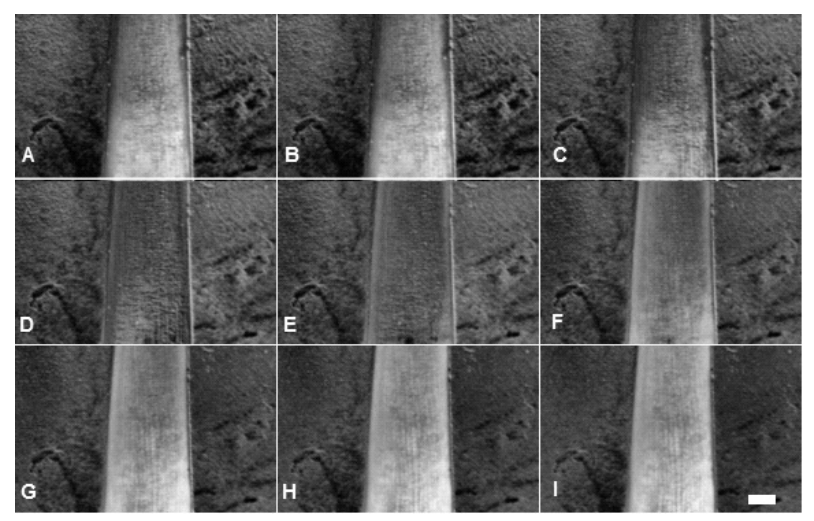

FIG. 6. DOLP images of the tendon under the illumination of the polarized light at $650 \pm 50 \mathrm{~nm}$ (red). Tendon was stretched with $30 \mathrm{MPa}$ of tensile stress. The IPA was rotated by $10^{\circ}$ from 10 to $90^{\circ}$ (A to I). The scale bar $=1 \mathrm{~mm}$. 
of the optical axes of the tendon, the micro-tear of the tendon was not shown well because of the strong linear polarization of the normal tendon. The DOLP image at the IPAs of $45^{\circ}$ (or $135^{\circ}$ ) most likely detected the micro tendon tear effectively, assuming that the fast axis of the tendon is parallel to the zero degree of IPA for the incident polarized light.

The partial-thickness tendon tears were relatively easily detectable by the regular CCD images under white light. However, the direction of the tendon tears affected the detection of the tears. When the direction of the tears was longitudinal and parallel to the orientation of the collagen fibers, the detection of the tears was not easy by the white CCD image (the CCD images in Figure 2). However, the DOLP images detected the partial thickness tears by representing the damages as the dark lines due to the loss of birefringence (Figure 2). While the CCD images under white light only depended on the reflected light intensity, the DOLP images detected the tendon tears in terms of DOLP and allowed more sensitive detection of the tears.

\section{Spectral imaging of tendon tears}

The incident polarized light of the micro tendon tears at different wavelengths of illuminating light allows the light penetration deep enough for the detection of subsurface damage. The utilization of visible RGB filters to the white light for the subsurface imaging allowed the light penetration at wavelengths ranging from 475 (blue) to 650 (red) $\mathrm{nm}$.

The polarization image of the tendon at the shorter wavelengths (blue or green) is formed by the light absorbed and scattered mostly from the surface. As the wavelength increases, the light penetrates more deeply and allows the polarization imaging at deeper depth. The image under blue light most likely displayed a surface scratch and the polarized images at $650 \mathrm{~nm}$ (red) revealed information of the tendon damage deeper in the tendon while polarized images under blue light provide information about more near-surface structures. The subtraction of the lower wavelength light image from the higher wavelength light image will lead to the image composed of the light reflected only from the deeper surface [10]. The subtraction will be conducted on the DOLP images of the turkey tendon in this study and the results will be reported separately.

\section{The effect of loading to tendon polarization property}

The increase of the DOLP with higher loading was reported in this study (Figure 3) and it is most likely due to better collagen fiber alignment with increasing tensile stress.

At tensile stresses higher than the yield point of the tendon, the tendon collagen fibers deform and will not return to their original shape. The damaged tendon in this study was shown as a dark area in the DOLP images. The breaking of the form birefringence of tendon changes was shown as the dark area in the DOLP images and it allowed the detection of the tendon tears (Figure 2-5).

The tendon was elongated as it was pulled by the stronger tensile stresses. However, the difference of the strains of tendons with tensile stresses over the yield point of the tendon should be small and the tensile force at the higher tensile stress will cause more damage to the tendon. In the Figure 1, it is believed that the tendons at the tensile stresses both 30 and $40 \mathrm{MPa}$ were damaged. However the tendon with the tensile stress of $40 \mathrm{MPa}$ seems to haveexperienced more severe damage of the tendon. In detail, the DOLP image of the Figure 1-D showed the overall damages of the tendon at the tensile stress of $40 \mathrm{MPa}$ while it was not shown in the tendon images at the lower tensile stresses (Figure 1-B and C).

\section{CONCLUSION}

The main advantages of the SPI system over other techniques such as CCD based arthroscopy, ultrasonography and MRI is its sensitivity to collagen fiber alignments. The polarization information can allow the detection of micro-tears of tendon as well as fullthickness tears by discriminating the fiber orientation. The rotating incident polarization angle (IPA) for the linearly polarized light provides a way to analyze different tissue types which may be sensitive to IPA variations. Arthroscopic diagnosis of micro and partialthickness tendon tear by utilization of stokes polarimetry imaging will be conducted to improve diagnostic capability.

\section{ACKNOWLEDGMENT}

This research was financially supported by the Ministry of Education, Science Technology (MEST), Koreas Science and Engineering Foundation(KOSEF, R01-2007-000-11427-0), Korea Institute for Advancement of Technology(KIAT) through the Human Resource Training Project for Regional Innovation.

\section{REFERENCES}

1. H. Ellman, "Diagnosis and treatment of incomplete rotator cuff tears," Clin. Orthop. Relat. Res. 254, 64-74 (1990).

2. S. K. Goergen, W. G. Bradley Jr., J. Liu, J. K. Tam, 
M. D. Dubin, P. J. Pema, L. M. Teresi, J. Jordan, and D. Morrison, "Improving the diagnostic accuracy of MR in the detection of infraspinatus tendon injuries," J. Comput. Assist. Tomogr. 20, 829-833 (1996).

3. S. A. Teefey, D. A. Rubin, W. D. Middleton, C. F. Hildebolt, R. A. Leibold, and K. Yamaguchi, "Detection and quantification of rotator cuff tears. Comparison of ultrasonographic, magnetic resonance imaging, and arthroscopic findings in seventy-one consecutive cases," J. Bone. Joint. Surg. Am. 86, 708-716 (2004).

4. J. H. Kim, J. Oh, T. E. Milner, and J. S. Nelson, "Hemoglobin contrast in magneto motive optical doppler tomography," Opt. Lett. 31, 778-780 (2006).

5. J. H. Kim, J. Oh, T. E. Milner, and J. S. Nelson, "Imaging nanoparticle flow using magneto-motive optical doppler tomography," Nanotechnology 18, 035504 (2007).

6. J. Oh, H. Lee, and J. Kim, "Detection of magnetic nanoparticles in tissue using magneto-motive DP-OCT," J. Opt. Soc. Korea 11, 26-33 (2007).
7. S. G. Demos and R. R. Alfano, "Optical polarization imaging," Appl. Opt. 36, 150-155 (1997).

8. S. G. Demos, A. J. Papadopoulos, H. Savage, A. S. Heerdt, S. Schantz, and R. R. Alfano, "Polarization filter for biomedical tissue optical imaging," Photochem. Photobiol. 66, 821-825 (1997).

9. P. J. Wu and J. T. Walsh, "Stokes polarimetry imaging of rat tail tissue in a turbid medium: degree of linear polarization image maps using incident linearly polarized light," J. Biomed. Opt. 11, 014031 (2006).

10. S. G. Demos, H. B. Radousky, and R. R. Alfano, "Deep subsurface imaging in tissues using spectral and polarization filtering," Opt. Exp. 7, 23-28 (2000).

11. W. B. B. Wang, J. H. Ali, R. R. Alfano, J. H. Vitenson, and J. M. Lombardo, "Spectral polarization imaging of human rectum-membrane-prostate tissues," IEEE J. Select. Topics Quantum Electron. 9, 288-293 (2003).

12. M. O'Brien, "Structure and metabolism of tendons," Scand. J. Med. Sci. Sports 7, 55-61 (1997). 